\title{
Interferometric synthetic aperture microscopy: tissue structure inferred by computed imaging techniques
}

\author{
Daniel L. Marks ${ }^{a}$, Tyler S. Ralston ${ }^{a}$, Brynmor J. Davis ${ }^{a}$, P. Scott Carney ${ }^{a}$, \\ and Stephen A. Boppart ${ }^{a, b}$ \\ ${ }^{a}$ Beckman Institute for Advanced Science and Technology and Department of Electrical \\ and Computer Engineering University of Illinois at Urbana-Champaign, \\ 405 N. Mathews, Urbana IL 61801 \\ ${ }^{b}$ Departments of Bioengineering and Medicine, University of Illinois at \\ Urbana-Champaign, Urbana IL 61801
}

\begin{abstract}
Interferometric Synthetic Aperture Microscopy (ISAM) ${ }^{1}$ is an optical microscopy computed-imaging technique for measuring the optical properties of three-dimensional structures and biological tissues. In this work, the principle of ISAM is reviewed, and its application to imaging tissue properties in various scanning geometries and instrument configurations is explored. The practicality of ISAM is demonstrated by imaging a rat heart and muscle using a real-time implementation of ISAM in conjunction with a clinical cart Optical Coherence Tomography instrument.
\end{abstract}

Keywords: Optical coherence tomography, inverse scattering

\section{AN INTRODUCTION TO ISAM}

Interferometric Synthetic Aperture Microscopy (ISAM) ${ }^{1}$ is an optical microscopy computed-imaging technique for measuring the optical properties of three-dimensional structures and biological tissues. It is related to optical coherence tomography ${ }^{2-4}$ (OCT) in that it utilizes interferometrically detected backwards-scattered light. However, unlike typical microscopy or OCT, ISAM can resolve the features that are outside of the depth-of-field of the microscope. Because resolving 3-D structure with a limited depth-of-field is a significant limitation of conventional optical microscopy, ISAM signficantly improves the ability of microscopy instruments to measure 3-D structure without scanning the focus. This advance has occurred because ISAM applies computed imaging techniques to coherence microscopy, rather than optically forming images. In this work, the principle of ISAM is reviewed, and its application to imaging tissue properties in various scanning geometries and instrument configurations $^{5-10}$ is explored.

OCT provides a unique means of sensing the micron-scale structure of tissues, however, one of its limitations is that the entire subject be inside the depth-of-field of the instrument. By using low

Further author information: (Send correspondence to S.A.B.)

S.A.B.: E-mail: boppart@uiuc.edu 
numerical aperture focusing, a high depth-of-field can be achieved at the expense of poorer transverse resolution. On the other hand, high numerical aperture tight focusing provides better transverse resolution, but the depth-of-field is small. While the focus may be scanned in the axial direction to mitigate this problem, this adds instrument complexity and slows down data acquisition. The problem of limited depth-of-field occurs because of the assumption that the illumination beam can simultaneously possess a narrow beam width and not diffract. While this assumption is sufficient when the wavelength is sufficiently short (as it is in X-ray tomography), in OCT the illumination wavelength is comparable in size to the desired resolution and therefore this assumption works poorly. When OCT was proposed, the digital signal processing methods needed to properly account for the the diffraction of the beam as ISAM does were probably not feasible in microcomputers and therefore the beam diffraction was neglected out of necessity. However, digital signal processing is now orders of magnitude faster and indispensable for methods such as swept source and spectral domain OCT. Because of this, methods such as ISAM are economically and practically feasible because the same signal processing hardware can be used that is already required for most spectral domain OCT instruments.

ISAM is a quantitative computational imaging method that infers object and tissue structure from interferometric ranging measurements. So what quantitative property of tissues does ISAM provide? ISAM measures the scattering of light off of tissue typically in the range of near infrared wavelengths from 700 to $1500 \mathrm{~nm}$. The electromagnetic properties that determine how light interacts with the medium are the magnetic and electric susceptibilities. In general, biological tissues are rather weakly diamagnetic, and therefore the scattering, absorption, and refraction are dominated by the electric susceptibility, and so only the electric susceptibility need be considered. Methods such as OCT and ISAM assume that the variation of susceptibility in the medium due to the structure is small, so that what is being measured is a small perturbation of the susceptibility added to a background of nearly constant susceptibility. This assumption works reasonably well though it can fail for highly scattering or refracting tissues. In addition, OCT and ISAM measure only the light that is backscattered from the sample. The backscattered light does not contain all information about the structure of the tissue. Measurements of backscatter primarily detect sharp boundaries and point-like objects, and are blind to areas with coarse structure. More specifically, OCT and ISAM can only measure high spatial frequencies of the object, and can not measure the lower spatial frequencies centered around the origin of the frequency space. Fortunately, many constituents of tissues, including cell nuclei, membranes, connective tissue, and other organelles are small and have sharp boundaries and therefore are detectable by OCT and ISAM. In summary, ISAM produces a quantitative reconstruction of the boundaries of the spatial variations of electric susceptibility that occur in tissues.

One of the chief improvements of ISAM over OCT is the ability of ISAM to computationally infer the susceptibility outside the depth-of-field. To explore how ISAM achieves this capability, we must first understand aperture synthesis. Aperture synthesis is more commonly known in the world of radar where it is used to produce synthetic aperture $\operatorname{radar}^{11,12}$ (SAR) images. SAR images are acquired by illuminating an object with radio pulses from many directions and detecting the reflected pulses off the object. Unlike OCT or ISAM where the beam is focused on the object, spotlight SAR images are acquired with a diverging beam, and individual features are not illuminated. Because the radio beam does not illuminate features sequentially, the radio signal cannot be examined directly to determine the features present in the illuminated scene. Instead, unlike OCT where the instrument operator can 
understand the data with no or little signal processing, SAR ranging data must be transformed into an image using computed tomographic techniques, because the raw data are projections of the image rather than the image itself. The data processing in SAR uses methods such as the Fourier projection slice theorem ${ }^{13}$ and the Fourier diffraction tomography theorem ${ }^{14}$ to transform the projection data into an image with a direct correspondence between samples in the image and features in the object. It is the diversity of the data acquired from many directions that enables SAR to resolve features inside the object despite the fact that the features are not individually illuminated by the radio beam.

Unlike SAR, OCT and ISAM do not acquire the data using a diverging beam. Rather, the sample is illuminated with a focused beam. In conventional OCT, the focus is usually scanned over all of the regions that one wishes to image, and the data acquired for which the object is out of focus is discarded. However, ISAM is able to use this discarded data to compute an image of the features outside of the focus. This is because as the beam is scanned, a point away from the focus is illuminated from many directions. This is illustrated in Fig. 1. In the figure, the beam is represented by curves representing the constant-time wavefronts as the beam converges to and diverges away from the focus. If a scatterer is in proximity of the focus, the beam only illuminates the scatterer for a short interval as the beam is translated laterally. Therefore the scatterer can be located to high resolution because of the narrow beam width at the focus. However, if the scatterer is away from the focus, the illumination beam is wide and therefore signal is returned for a much larger interval of the translation of the beam. Therefore it would appear that the scatterer cannot be located as well because of the "smearing" effect produced by the wider beam. However, the focused beam itself contains components that are traveling in many directions, all pointed toward or away from the focus. As the beam is translated, the different parts of the illumination beam are incident on the scatterer, each traveling in a slightly different direction. This is how the diversity of illumination directions occurs, in a manner similar to SAR. A focused beam itself contains a diversity of directions that are used in ISAM to synthesize the image outside of the focus. However, a somewhat different algorithm is needed to transform the data into an image for ISAM because a focused, rather than a diverging beam is used.

While SAR and ISAM have very different ways of sampling the structure of the object, the algorithms used to synthesize the image from the projection data have a common mathematical structure. One common way that the image processing is implemented in SAR and X-ray Computed Tomography is to use the Fourier projection-slice theorem. This theorem is implemented typically as a change of the data from polar to rectangular coordinates in the Fourier spatial-frequency space. For discretely sampled data, a change of coordinates is called a resampling and is the recreation of a function that is sampled uniformly in one set of coordinates to one that is sampled uniformly in a different set of coordinates. This appears as a "warping" or "stretching" of the resampled function onto the new coordinate system. The mathematical operation in ISAM that compensates for the defocus is a resampling as it is in SAR, but using a different transformation between coordinates. Those who are already familiar with SAR or Computed Tomography signal processing likely are well prepared to implement ISAM because of the similarity between the signal processing methods.

As a simple example of the improvement ISAM is capable of, Fig. 2 presents an image of a collection of pointlike scatterers before and after ISAM signal processing. The object is titanium dioxide particles of $2 \mu \mathrm{m}$ diameter embedded in cured transparent polydimethylsiloxane polymer. Without ISAM processing, the left panel (a) shows a degradation of resolution toward the top of the 

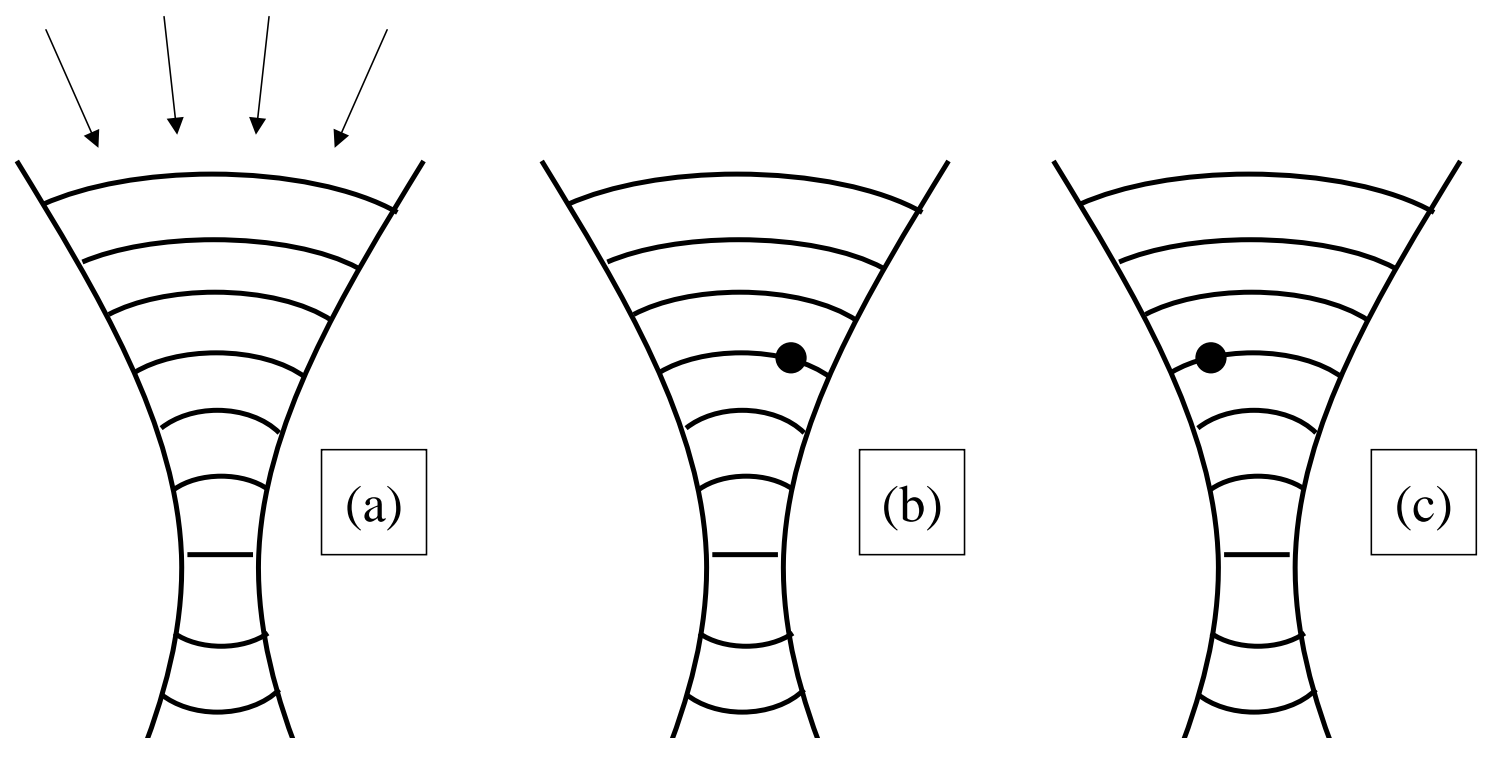

Figure 1. Illustration of a how a focused beam contains a diversity of directions. The arrows of part (a) that point toward the focus show the directions in various parts of the wave front. Parts (b) and (c) show how as the beam is translated laterally relative to a scatterer, different parts of the wavefront scatter, providing the diversity of reflected directions in the reflected signal. 
image, whereas the focus is at the bottom of the image. The resolution is made uniform throughout the image in the right panel (b) after ISAM processing. For reference, the dimensions of both images are $500 \mu \mathrm{m}$ (transverse) by $1000 \mu \mathrm{m}$ (axial), with a confocal parameter is $68 \mu \mathrm{m}$. The insets allow detailed study of the reconstructions of individual scatterers which are in the dotted boxed areas of the respective images. In the left inset, one observes interference between the fields from individual scatterers as a classic stripe or dotted-line pattern. This is a signature of the coherent defocus of OCT, and is what allows ISAM to be performed. These interference patterns are not present in the right inset after ISAM processing as the pointlike scatterers are individually resolved and interference effects do not occur. 

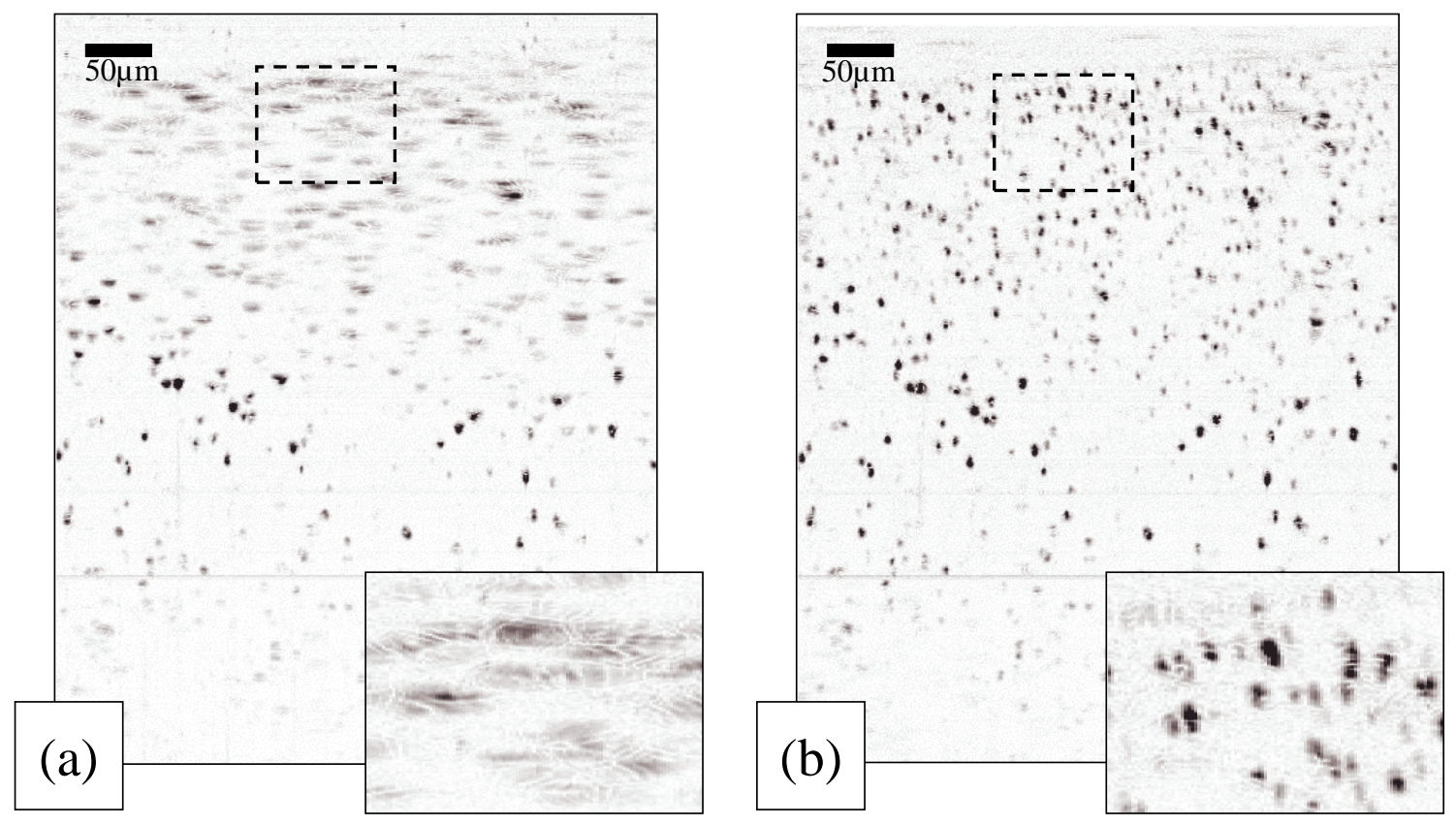

Figure 2. An example showing how ISAM improves the image of a simple object consisting of titanium dioxide particles embedded in a silicone medium. Part (a) is the "raw" OCT image data, which shows the effect of defocus towards the top of the image. Part (b) shows the ISAM corrected image, which properly accounts for the defocus of the beam. The insets show a magnifi ed region at the top of the image that exemplifi es the improvement ISAM produces. 


\begin{tabular}{|c|c|c|c|c|}
\hline \multicolumn{5}{|c|}{ ISAM Methods and Applicable Geometries } \\
\hline Algorithm Type & Beam Type & Scanning Type & Resampling & Comments \\
\hline $\begin{array}{l}\text { Low Numeri- } \\
\text { cal Aperture }\end{array}$ & $\begin{array}{l}\text { Focused } \\
\text { Paraxial } \\
\text { Gaussian } \\
\text { Beam } \\
(N A \\
0.1)\end{array}$ & $\begin{array}{l}\text { Lateral Trans- } \\
\text { lation Scan }\end{array}$ & $\begin{array}{l}\text { Paraxial } \\
\text { Stolt }\end{array}$ & $\begin{array}{l}\text { For most conventional opti- } \\
\text { cal coherence tomography se- } \\
\text { tups, scanning the focus over } \\
\text { a plane }\end{array}$ \\
\hline $\begin{array}{l}\text { High Numeri- } \\
\text { cal Aperture }\end{array}$ & $\begin{array}{l}\text { Focused } \\
\text { Non- } \\
\text { Paraxial } \\
\text { Gaussian } \\
\text { Beam } \\
\text { (arbitrary } \\
N A<1.0)\end{array}$ & $\begin{array}{l}\text { Lateral Trans- } \\
\text { lation Scan }\end{array}$ & Stolt $^{15}$ & $\begin{array}{l}\text { For optical coherence mi- } \\
\text { croscopy, scanning the focus } \\
\text { over a plane }\end{array}$ \\
\hline $\begin{array}{l}\text { Rotational } \\
\text { Scan }^{7}\end{array}$ & $\begin{array}{l}\text { Focused } \\
\text { Gaussian } \\
\text { Beam } \\
(N A \\
1.0)\end{array}$ & $\begin{array}{l}\text { One dimen- } \\
\text { sion rotation, } \\
\text { one dimension } \\
\text { translation }\end{array}$ & $\begin{array}{l}\text { Stolt / } \\
\text { Polar to } \\
\text { Rectan- } \\
\text { gular }\end{array}$ & $\begin{array}{l}\text { For catheter-based OCT in- } \\
\text { struments, scanning the focus } \\
\text { over a cylinder }\end{array}$ \\
\hline $\begin{array}{l}\text { High NA Vec- } \\
\text { tor }^{10}\end{array}$ & $\begin{array}{l}\text { Focused } \\
\text { Non- } \\
\text { Paraxial } \\
\text { Beam } \\
\text { (arbitrary } \\
N A<1.0 \text { ) } \\
\end{array}$ & $\begin{array}{l}\text { Lateral Trans- } \\
\text { lation Scan }\end{array}$ & Stolt & $\begin{array}{l}\text { Used when one wishes to ac- } \\
\text { count for vector nature of } \\
\text { illumination and reconstruct } \\
\text { components of susceptibility } \\
\text { tensor of object }\end{array}$ \\
\hline Full-Field $^{8}$ & Plane Wave & $\begin{array}{l}\text { Wavelength } \\
\text { Scan }\end{array}$ & $\begin{array}{l}\text { Fourier } \\
\text { Diffrac- } \\
\text { tion } \\
\text { Tomog- } \\
\text { raphy }\end{array}$ & $\begin{array}{l}\text { For full-field optical coher- } \\
\text { ence tomography without } \\
\text { scanning the focus }\end{array}$ \\
\hline $\begin{array}{l}\text { Full-Field Par- } \\
\text { tially Coherent } \\
\text { Illumination } \\
\text { (in prepara- } \\
\text { tion) } \\
\end{array}$ & $\begin{array}{l}\text { Schell- } \\
\text { Model } \\
\text { Partially } \\
\text { Coherent } \\
\text { Source }\end{array}$ & $\begin{array}{l}\text { Wavelength } \\
\text { Scan }\end{array}$ & Hybrid & $\begin{array}{l}\text { Used for partially coherent il- } \\
\text { lumination in full-field mi- } \\
\text { croscopy, which is common }\end{array}$ \\
\hline
\end{tabular}

Table 1. Summary of ISAM algorithms and their geometries.

\section{ISAM GEOMETRIES}

To apply ISAM to the data acquired by a particular instrument, one must understand the various types of ISAM algorithms available and the geometries that these apply to. These geometries are given 


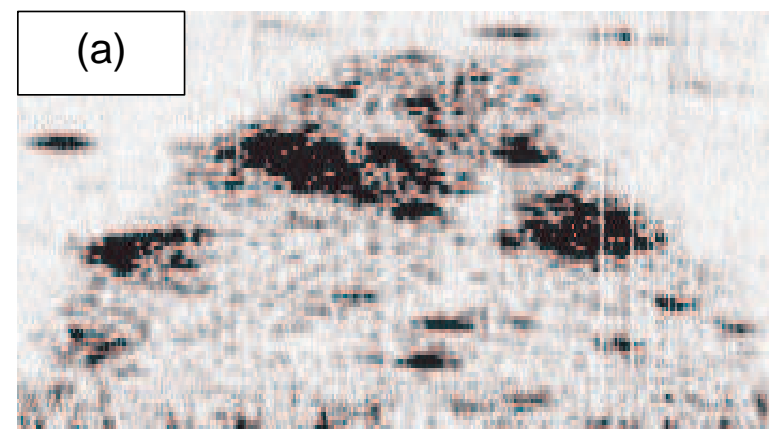

$1.6 \mathrm{~mm} \mathrm{H} \times 1.0 \mathrm{~mm} \mathrm{~V}$

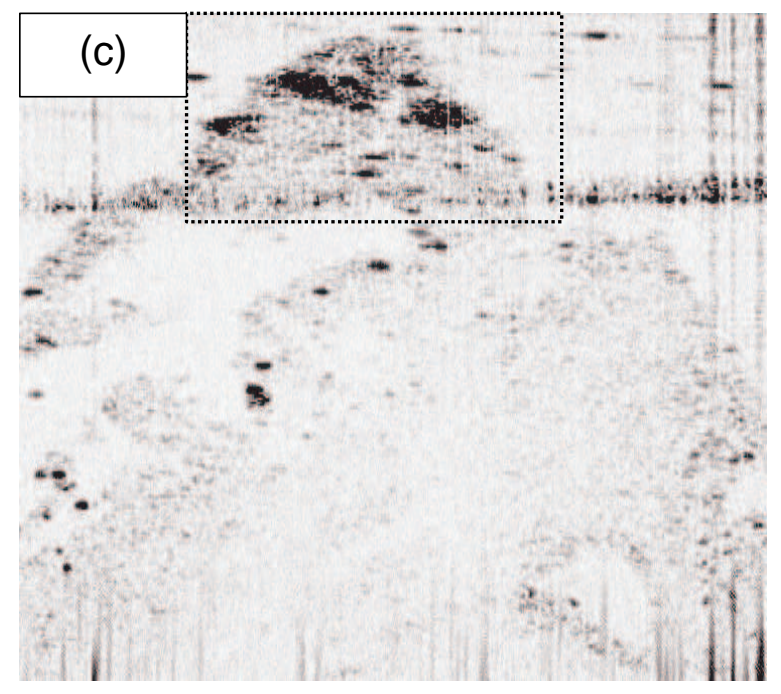

$3.5 \mathrm{~mm} \mathrm{H} \times 3.5 \mathrm{~mm} \mathrm{~V}$

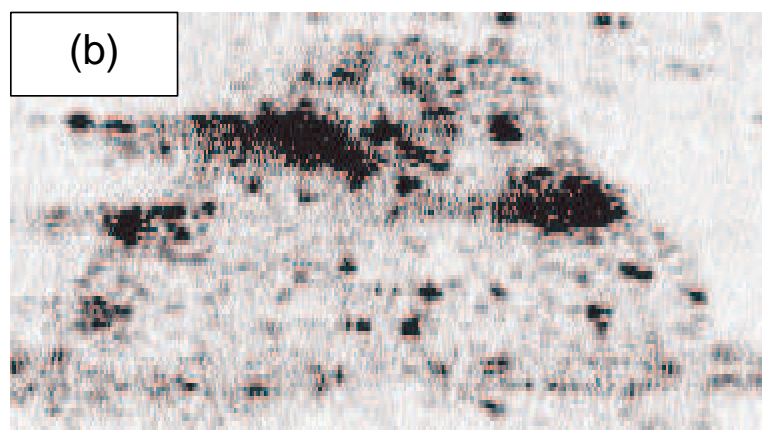

$1.6 \mathrm{~mm} \mathrm{H} \times 1.0 \mathrm{~mm} \mathrm{~V}$

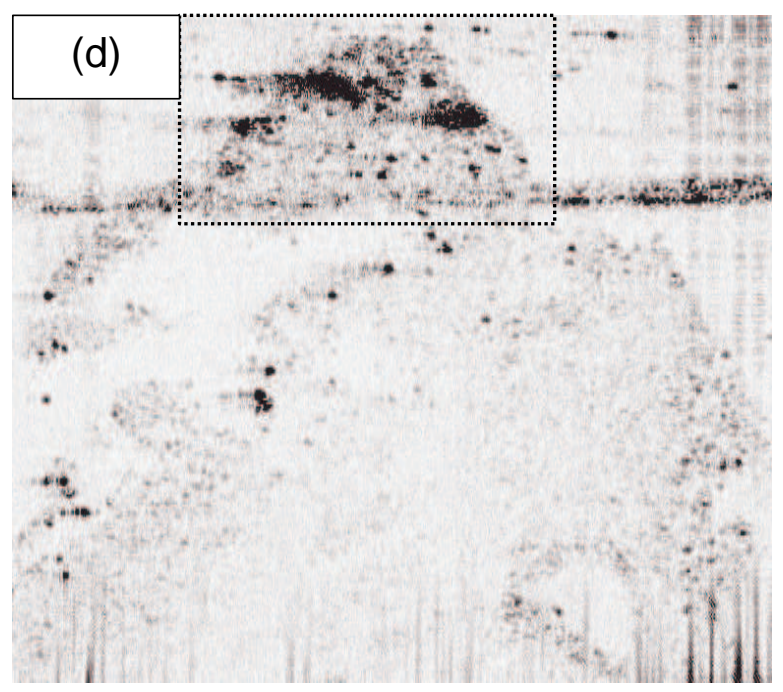

$3.5 \mathrm{~mm} \mathrm{H} \times 3.5 \mathrm{~mm} \mathrm{~V}$

Figure 3. An image of a rat heart taken without (a and c) and with (b and d) ISAM on the clinical instrument. Parts (a) and (b) are magnifi ed portions of the total image, before (a) and after (b) ISAM. The improvement of ISAM is manifested by scatterers becoming more pointlike. Parts (c) and (d) are the entire image before (c) and after (d) ISAM processing. Near the bottom where the focus is, there is little change to the image. However, as one approaches the top further from the focus the improvement becomes signifi cant.

by the way the illumination beam is scanned and the shape of the illumination beam. Table 1 is a summary of the extant ISAM algorithms, the beam type and scanning types, and the description of the application of the algorithm. Note that the high numerical aperture methods are applicable to low numerical aperture instruments. The two main categories are scanned beam and full-field. The scanned beam case has been solved for both low and high numerical apertures, and recently for the vector-diffraction case accounting for the polarization of the incident beam and a general dielectric tensor object. 


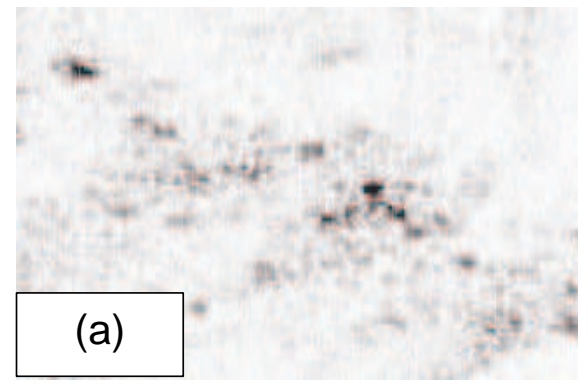

$1.5 \mathrm{~mm} \mathrm{H} \times 1.0 \mathrm{~mm} \mathrm{~V}$

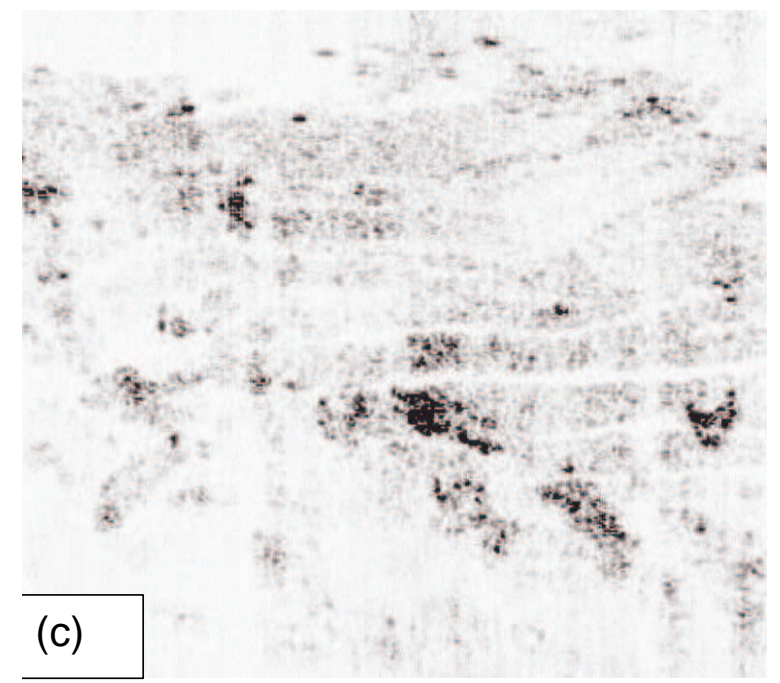

$3.9 \mathrm{~mm} \mathrm{H} \times 3.5 \mathrm{~mm} \mathrm{~V}$

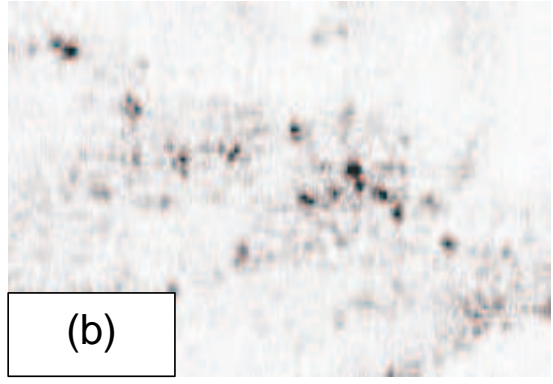

$1.5 \mathrm{~mm} \mathrm{H} \times 1.0 \mathrm{~mm} \mathrm{~V}$

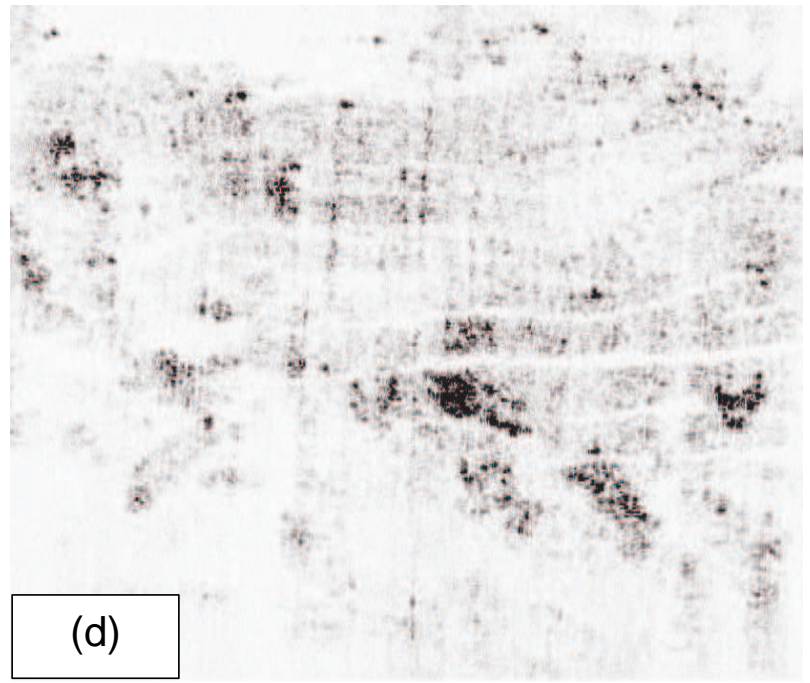

$3.9 \mathrm{~mm} \mathrm{H} \times 3.5 \mathrm{~mm} \mathrm{~V}$

Figure 4. An image of rat muscle taken without (a and c) and with (b and d) ISAM on the clinical instrument. Parts (a) and (b) are magnifi ed portions of the total image, before (a) and after (b) ISAM. The improvement of ISAM is manifested by scatterers becoming more pointlike. Parts (c) and (d) are the entire image before (c) and after (d) ISAM processing. Near the bottom where the focus is, there is little change to the image. However, as one approaches the top further from the focus the improvement becomes signifi cant. 


\section{REAL-TIME ISAM IN CLINICAL APPLICATIONS}

ISAM $^{1}$ is very amenable to implementation using digital signal processing hardware, especially the multicore and multiprocessor architectures being developed by IBM, Intel, Nvidia, and Advanced Micro Devices for the next generation of personal computers and home entertainment devices. Exploiting the computational power and ubiquity of consumer electronics-level hardware is essential to achieve the cost and portability requirements needed for a mobile clinical instrument. Fortunately, similar digital signal processing techniques as those used in ISAM are already widely employed in cellular telephones, video decompression and processing hardware, and three-dimensional visualization. In particular, the primarily numerical operations in ISAM that constitute most of the computation are one-dimensional Fast Fourier Transforms and one-dimensional resampling operations. During the ISAM computation, these operations are performed on separate portions of the data set, such as on individual axial scans. This provides a natural way to distribute the computation between multiple processors so that each processor performs a one-dimensional computation to a portion of the data set.

As a step to produce a high-speed implementation of ISAM for the clinic, ISAM was implemented in $\mathrm{C}++$ for a clinical cart optical coherence tomography instrument. The source was a superluminescent diode at $1300 \mathrm{~nm}$ center wavelength and $80 \mathrm{~nm}$ full-width-half-maximum bandwidth. The OCT system used a beam scanned by galvanometer-actuated mirrors, for which the planar-scan high-numerical-aperture ISAM algorithm was used. This algorithm was implemented on a dual-core Athlon X2 4400 processor computer with 2 GB RAM. A Sensors Unlimited InGaAs 1024 element photodiode array detected the intensity of the sampled spectrum. Each image consisted of $M=1000$ axial scans.

To evaluate the computational burden imposed by ISAM, we consider that the standard spectraldomain and frequency swept-source OCT methods typically require two operations for each axial scan: a resampling of the acquired spectrum to be linear in the spatial frequency of the sample medium, and an real-to-complex Fourier transform to compute the spatial distribution of scatters along the axial scan. In the clinical OCT system, the resampling of each axial scan was performed using a approximate prolate spheroidal sampling interpolator. ${ }^{16}$ The benefit of this interpolator is that it is able to achieve high interpolation accuracy for signals sampled near Nyquist without upsampling the data. Our interpolator was designed with 9 taps for a signal of $75 \%$ of Nyquist, which is given by the following formula:

$$
y_{n}=\sum_{k=\operatorname{round}(n)-T}^{\operatorname{round}(n)+T} x_{k} \frac{\sin \pi(n-k)}{\pi(n-k)} \frac{\sinh \left[c \sqrt{1-\left(\frac{n-k}{T}\right)^{2}}\right]}{(\sinh c) \sqrt{1-\left(\frac{n-k}{T}\right)^{2}}}
$$

where $x_{k}$ is the sampled spectrum on the camera, $y_{n}$ is the resampled spectrum, $k$ are the indices of the original spectrum (from 0 to 1023), and $n$ are the indices of the resampled spectrum. The round function refers to rounding to the nearest integer. The variable $c=\pi T \delta$ is the space-bandwidth product of the sampled signal, and $1-\delta$ is the fraction of Nyquist that the original signal is bandlimited to. In this case, $\delta=0.25$ for a signal $75 \%$ of Nyquist. A good choice of $T$ is $1 / \delta$, so that the filter 
has $2 T+1$ taps, so that in this case $T=4$ for 9 taps. In our case, the taps for each point $t$ were precomputed and stored in RAM for maximum speed.

The forward and inverse Fast Fourier transforms were performed using the FFTW library, a freesoftware based library that is optimized for speed using single-instruction-multiple-data (SIMD) instructions for many architectures, especially Intel SSE1/2/3 for the IA-32 architecture. The ISAM implementation used a single-precision IEEE 754 floating point number representation because the accuracy was sufficient and the speed was much improved over the double-precision implementation.

For the spectral-domain imaging without ISAM, the acquisition rate was about 2 frames per second at an axial scan sampling rate of $8000 \mathrm{~Hz}$. The ISAM implementation adds several additional steps to the calculation. In our version of ISAM, we chose to add digital dispersion compensation ${ }^{17}$ to help remove effects of unbalanced dispersion in the interferometer. Especially when very large bandwidths are used, digital dispersion compensation becomes attractive even at the expense of additional calculation because optical compensation is increasingly difficult. The steps for the ISAM calculation follow:

1. First the real-valued sampled spectrum $x_{k}$ (for $k$ from 0 to $N-1$, with $N=1024$ ) for each of the $M$ axial scans was resampled to be linear spatial frequency as $y_{n}$ using the interpolator Eq. 1.

2. The one-dimensional real-to-complex FFT was computed to find the samples of the complex spatial-domain signal (of 512 complex-valued samples) from each axial scan spectrum, equivalent to:

$$
Y_{t}=\sum_{n=0}^{N-1} y_{n} \exp (-2 \pi i t n / N)
$$

for $t$ from 0 to $N / 2-1$. This calculation is proper only if one assumes that the real-valued spectrum is the real part of a complex analytic signal. The sampled spectrum will be a complex analytic signal if the reference signal in the interferometer completely precedes the sample signal in time when the two are recombined at the beam splitter.

3. The spatial-domain signal $Y_{t}$ is padded with $N / 2$ zeros on the right (high frequency) end to double the length of the signal, so $Y_{t}$ consists of 1024 complex-valued samples and $t$ spans from 0 to $N-1$.

4. The one-dimensional complex-to-complex FFT is taken to produce an upsampled version of the spectrum $\tilde{y}_{n}$ (with 1024 complex-valued samples) which now has both real and imaginary parts corresponding to the full complex analytic signal.

5. The spectrum $\tilde{y}_{n}$ is multiplied by a filter to form $\tilde{y}_{n}^{\prime}$ :

$$
\tilde{y}_{n}^{\prime}=\tilde{y}_{n} \exp \left[-\frac{\pi i}{N}\left(2 \tau\left(n-n_{0}\right)+\alpha_{2}\left(n-n_{0}\right)^{2}+\alpha_{3}\left(n-n_{0}\right)^{3}\right)\right]
$$

The purpose of this filter is twofold. First, it corrects the interferometer dispersion with a proper choice of $\alpha_{2}$ and $\alpha_{3}$. More importantly, it translates the axial scan so that the region of each 
axial scan that corresponds to the focus of the beam is placed at the sample $n=1$. Therefore one must know the sample number $\tau$ which corresponds to the delay at which the beam is focused. The value $n_{0}$ corresponds to the sample number of the spectrum where the spectrum is centered.

6. The one-dimensional complex-to-complex FFT is taken of all of the axial scans $\tilde{y}_{n}^{\prime}$ across the direction of the $M$ axial scans, as opposed to along the axial scans as performed earlier. This data will be called $\tilde{y}_{n, m}^{\prime}$ where $n$ is the index corresponding to the axial spatial frequency (with $n$ from 0 to $N-1$ ) and $m$ corresponding to transverse spatial frequency (with $m$ from $-M / 2$ to $M / 2-1)$. Note that most FFT implementations place positive frequencies first, then negative frequencies afterwards.

7. A set of one-dimensional resampling operations is now performed to the data $\tilde{y}_{n, m}^{\prime}$ along the $n$ direction to compute a new array $\tilde{y}_{n^{\prime}, m}^{\prime \prime}$. The indices of the points $n^{\prime}$ to resample in $\tilde{y}_{n, m}^{\prime}$ are given by:

$$
n^{\prime}=\sqrt{m^{2} \beta^{2}+n^{2}}
$$

where $\beta$ is a proportionality constant between the scale of the axial and transverse spatial frequencies. A prolate-spheroidal interpolator with precomputed taps was used to resample in this step.

8. A one-dimensional inverse complex-to-complex FFT was performed to data set $\tilde{y}_{n^{\prime}, m}^{\prime \prime}$ along the direction of $m$ to compute ISAM-corrected spectra $\tilde{y}_{n^{\prime}}^{\prime \prime}$.

9. Each axial scan $\tilde{y}_{n^{\prime}}^{\prime \prime}$ is translated to put the focus back at the correct position in the image:

$$
\tilde{y}_{n^{\prime}}^{\prime \prime \prime}=\tilde{y}_{n^{\prime}}^{\prime \prime} \exp \left[\frac{\pi i}{N}\left(2 \tau\left(n^{\prime}-n_{0}\right)\right)\right]
$$

10. The one-dimensional inverse complex-to-complex transform was performed to the arrays $\tilde{y}_{n^{\prime}}^{\prime \prime \prime}$ along the $n^{\prime}$ direction to yield to the final image $\tilde{Y}_{t}$.

As can be readily seen, ISAM adds a significant amount of computation (steps $3-10)$. However, both dispersion compensation and ISAM are performed, and the additional computation is $O(N \log N)$ in the data set size, so that the ISAM computation asymptotically scales identically to normal SD-OCT signal processing. With all the additional processing, the clinical cart can image at one frame per second at $8000 \mathrm{~Hz}$ axial scan rate.

Examples of imaging with the clinical cart are presented in Figures. 3 and 4. These were of rat heart and muscle, preserved in a special optical clearing agent so that the beam penetration would be large. As a result, the penetration was over four times the penetration achievable on untreated tissue, and the scattering from the samples was exceptionally low. Because of the weak scattering, the imaging was performed at $300 \mathrm{~Hz}$ axial scan rate to achieve a satisfactory signal-to-noise ratio. The numerical aperture of the focused illumination was 0.03 so that the depth of field was very large, which required a long axial scan range to achieve noticeable defocus. The focus was toward the bottom of each image, so that the greatest defocus appears near the surface of each of the tissues. 
In each case, pointlike scatterers that are poorly resolved in the image without ISAM processing are resolved and sharp in the ISAM processed image. In addition, ISAM helps resolve the banding structure in the muscle. The ISAM processing for both of these images was performed in real time in about one second after the three second acquisition time of each image. Therefore ISAM signal processing did not add greatly to the total time needed for image acquisition. As can be seen, ISAM can produce significantly better imagery of tissue structures at practical frame rates on commodity computer hardware without optimization. In the future, the code can be optimized for multicore and SIMD architectures and the speed can be further improved. ISAM is indeed coming into its own as a practical means of image enhancement for coherence-ranging based optical instruments.

\section{Acknowledgments}

This work was supported by a Grainger Foundation grant and the National Institutes of Health (NIH NIBIB 1 R01 EB005221).

\section{REFERENCES}

1. T. S. Ralston, D. L. Marks, P. S. Carney, and S. A. Boppart, 'Interferometric synthetic aperture microscopy," Nat. Phys. 5, pp. 129-134, 2007.

2. J. A. Izatt, H.-W. Kulkarni, K. Wang, M. W. Kobayashi, and M. W. Sivak, 'Optical coherence tomography and microscopy in gastrointestinal tissues,' IEEE J. Selected Topics in Quantum Elect. 2, pp. 1017-1028, 1996.

3. J. G. Fujimoto, S. A. Pitris, S. A. Boppart, and M. E. Brezinski, 'Optical coherence tomography: an emerging technology for biomedical imaging and optical biopsy,"Neoplasia 2, pp. 9-25, 2000.

4. S. A. Boppart, B. E. Bouma, C. Pitris, J. F. Southern, M. E. Brezinski, and J. G. Fujimoto, 'In vivo cellular optical coherence tomography imaging," Nature Medicine 4, pp. 861-864, 1998.

5. T. S. Ralston, D. L. Marks, P. S. Carney, and S. A. Boppart, 'Inverse scattering for optical coherence tomography," J. Opt. Soc. Am. A 23, pp. 1027-1037, 2006.

6. T. S. Ralston, D. L. Marks, S. A. Boppart, and P. S. Carney, 'Inverse scattering for high-resolution interferometric microscopy," Opt. Lett. 31, pp. 3585-3587, 2006.

7. D. L. Marks, T. S. Ralston, P. S. Carney, and S. A. Boppart, "Inverse scattering for rotationally scanned optical coherence tomography," J. Opt. Soc. Am. A 23, pp. 2433-2439, 2006.

8. D. L. Marks, T. S. Ralston, S. A. Boppart, and P. S. Carney, 'Inverse scattering for frequency-scanned full-fi eld optical coherence tomography," J. Opt. Soc. Am. A 24, pp. 1034-1041, 2007.

9. B. J. Davis, T. S. Ralston, D. L. Marks, S. A. Boppart, and P. S. Carney, "Autocorrelation artifacts in optical coherence tomography and interferometric synthetic aperture microscopy," Opt. Lett. 32, pp. 14411443, 2007.

10. B. J. Davis, S. C. Schlachter, D. L. Marks, T. S. Ralston, S. A. Boppart, and P. S. Carney, 'Nonparaxial vector-fi eld modeling of optical coherence tomography and interferometric synthetic aperture microscopy," J. Opt. Soc. Am. A 24, pp. 2527-2542, 2007.

11. D. E. Wahl, P. H. Eichel, D. C. Ghiglia, P. A. Thompson, and C. V. Jakowatz, Spotlight-Mode Synthetic Aperture Radar: A Signal Processing Approach, Springer, New York, 1996.

12. J. C. Curlander and R. N. McDonough, Synthetic Aperture Radar: Systems and Signal Processing, Wiley, New York, 1991. 
13. F. Natterer, The Mathematics of Computerized Tomography, Society for Industrial Mathematics, Philadelphia, 2001.

14. A. C. Kak and S. Slaney, Introduction to Inverse Problems in Imaging, Society for Industrial Mathematics, Philadelphia, 2001.

15. R. H. Stolt, 'Migration by fourier transform," Geophys. 43, pp. 23-48, 1978.

16. J. J. Knab, 'Interpolation of band-limited functions using the approximate prolate series," IEEE Trans. on Information Theory IT-25, pp. 717-720, 1979.

17. D. L. Marks, A. L. Oldenburg, J. J. Reynolds, and S. A. Boppart, "A digital algorithm for dispersion correction in optical coherence tomography for homogeneous and stratifi ed media," Appl. Opt. 42, pp. 204217, 2003. 